\title{
Multi-Order Intermittent Chaotic Synchronization of Closed Phase Locked Loop
}

\author{
Samir M. Shariff \\ Electrical Engineering Department, College of Engineering, Taibah University, Medinah, KSA \\ Email: samshariff@yahoo.com
}

How to cite this paper: Shariff, S.M. (2018) Multi-Order Intermittent Chaotic Synchronization of Closed Phase Locked Loop. International Journal of Modern Nonlinear Theory and Application, 7, 48-55. https://doi.org/10.4236/ijmnta.2018.72004

Received: December 4, 2017

Accepted: June 9, 2018

Published: June 12, 2018

Copyright $\odot 2018$ by author and Scientific Research Publishing Inc. This work is licensed under the Creative Commons Attribution International License (CC BY 4.0).

http://creativecommons.org/licenses/by/4.0/

\begin{abstract}
For the model of a Closed Phase Locked Loop (CPLL) communication System consists of both the transmission and receiver ends. This model is considered to be in a multi-order intermittent chaotic state. The chaotic signals are then synchronized along side with our system. This chaotic synchronization will be demonstrated and furthermore, a modulation will be formed to examine the system if it will perfectly reconstruct or not. Finally we will demonstrate the synchronization conditions of the system.
\end{abstract}

\section{Keywords}

Chaotic Synchronization, Chaotic Signal, Communication Systems, Closed Phase Locked Loop System, Multi-Order Model

\section{Introduction}

A chaotic system has various properties one of which we will consider in our paper here is the property of uncorrelated trajectory that forms by the exponential divergence of the initial conditions, which varies with time. It is not straightforward to demonstrate a pair of chaotic systems that get synchronized together perfectly.

In previous work done by Pecora and Carroll [1] [2] [3], an interesting result was produced in exploring the properties of chaos synchronization for nonlinear systems and its applications. Additional research was conducted in the area of secure communications in particular for a phase locked loop model.

Our model's state variables are time variant composed of a set of pair differential equations for each identical system that is in a chaotic state.

The eminent theory of synchronization contains in the deterministic concept of chaotic systems. With time variation, the deterministic system of the transition of the state variables is connected by a given set of differential equations. In 
our case, two identical chaotic systems are assumed to be simultaneously active. Hence, the differential equations will govern the state variables of each system. Moreover, the set of the initial conditions is reflected by the divergence of the trajectories that were initially stated.

In this paper, the use of a closed phase locked loop CPLL system is studied. This system consists of a transmission and receiver that will be forced by a common chaotic signal. Furthermore, the system representing the mathematical model was derived of the differential equation and then was simulated. These results will be developed through the synchronization of the multi-order intermittent CPLL model [4] [5] [6] [7].

\section{Cpll Model}

Chaos in closed phase locked loops has been researched by many researchers in various institutions around the world for at past few years. Closed phase locked loops similar to many chaotic systems that are close to Chua's circuit series [8], Josephson junctions [9] and Van der Pol oscillator [10] have been considered extensively in this work. A more recent study was made on chaos in closed phase locked loop has stretched beyond analysis of chaotic behavior. The general notion of chaos synchronization was used to build a communication system that will ensure the security of information transmitted with it.

The CPLL that was considered as a chaos generator for this systems has a response that is undesirable for many of the typical communication systems. In one case, the CPLL is used to demodulate an FM signals, as well as the output of the system may become chaotic for particular loop parameters. This behavior drives the CPLL to reach to unlock its state [11]. In both cases, chaos observation and control, for the undesirable state, are of a vital interest for this design parameters.

Both, Endo and Watada [12] found that the bifurcation sets of the Shilinkov-type homoclinic orbits for a third-order independent CPLL with a symmetric periodic triangular phase detector that the filter is in a second order loop. It is clear that this type of CPLL has a chaotic attractor. Concurrently, both, Harb \& Harb [13] presented a third order CPLL with sinusoidal phase detector characteristics experienced chaos through Hopf bifurcation. In addition, the CPLL is unlocked after the Hopf bifurcation point was indicated. Both bifurcation and chaos theory were analyzed based on modern nonlinear control theory and methodologies. Both methods of multi scales, and perturbation, have been analyzed to determine the normal form at the surroundings of the Hopf bifurcation focal point. This point was considered to be supercritical. Such that, a small periodic solutions, limit cycles, are originated at the Hopf bifurcation point. The increase of the control parameter will limit the cycle's deformity as well as eventually ending to reach to a chaotic state.

Finally, both Harb \& Harb [14], considered the closed phase locked which block circuit diagram is presented in Figure 1. The loop filter is represented as, 


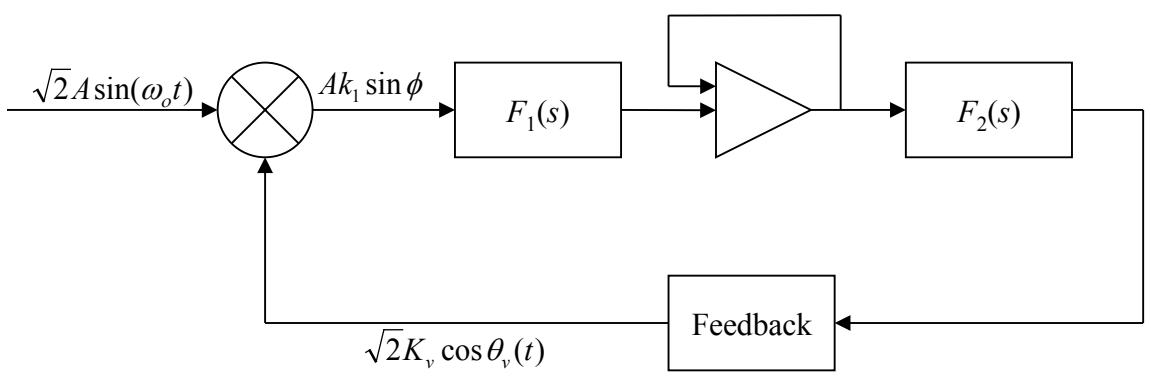

Figure 1. Block diagram of a closed phase locked loop with second-order loop filter and feedback.

Next we superimpose the control signal into the CPLL system, and then integrate it into the new system form; this will enable it to obtain the simulation results as illustrated in Figure 3. In Figure 2 it is obvious that the chaotic behavior through Hpof bifurcation is uncontrolled. Since the control parameter Ko is around 85300 where the chaotic oscillation present a double rout to the current chaos. Thus, the CPLL is forced to reach a state of out of lock and will not reach to a state of equilibrium, phased locked. The main aim of this work is to reach a controlled equilibrium state clearing all chaotic behaviors. Now, looking at both figures in Figure 2 and in Figure 3, we correlate them and notice that it will approach a stable equilibrium state solution. This indicates that the CPLL in a lock state. Furthermore, we undertake the design of the control signal that is taken after 50 seconds from the initial time of origination. Finally, Figure 4 presents the controller as it takes the system to a stable equilibrium state solution in a locked state of the original control signal.

The closed phase locked loop model is sketched in the block diagram shown in Figure 5. This model will be investigated for chaotic synchronous pair attractors. Our model consists of a multi order system. We will denote it by n-CPLLs, and for simplicity we will take a 3rd order system. Thus we will have 3-CPLLs that we will indicate them as follows, 0-CPLL will be a positive Lyaponouv exponent. This 0-CPLL will generate a chaos for its VOC output that will be applied to both inputs of 1-CPLL and 2-CPLL systems. Both 1-CPLL and 2-CPLL have a negative Lyaponouv exponent and are driven by a common chaotic signal $c(t)[15]$.

We have a chaotic 1-CPLL that will drive the complete system to be at state of out of lock. Thus, this will convert the receiver chaotic signal of 0-CPLL to produce anther chaotic situation. Meanwhile, 2-CPPL will in turn becomes an attractor of chaos, and the common chaotic signal $\mathrm{c}(\mathrm{t})$ will synchronize the complete system. We note that the initial conditions and parameters are unchanged and are identical throughout the numerical solution [16].

We then modulate the information signal $\mathrm{m}(\mathrm{t})$ in the transmission end of the system with a chaotic signal. Furthermore, we then synchronize the receiver end of the system along with the transmission end of the system. Figure 2 shows that both the receiver and transmitter ends of our system are identical. 


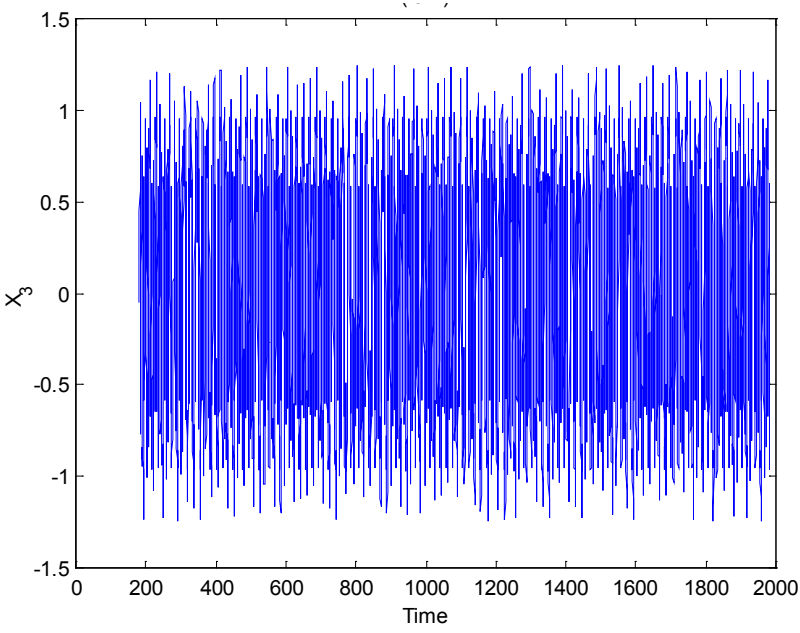

(a)

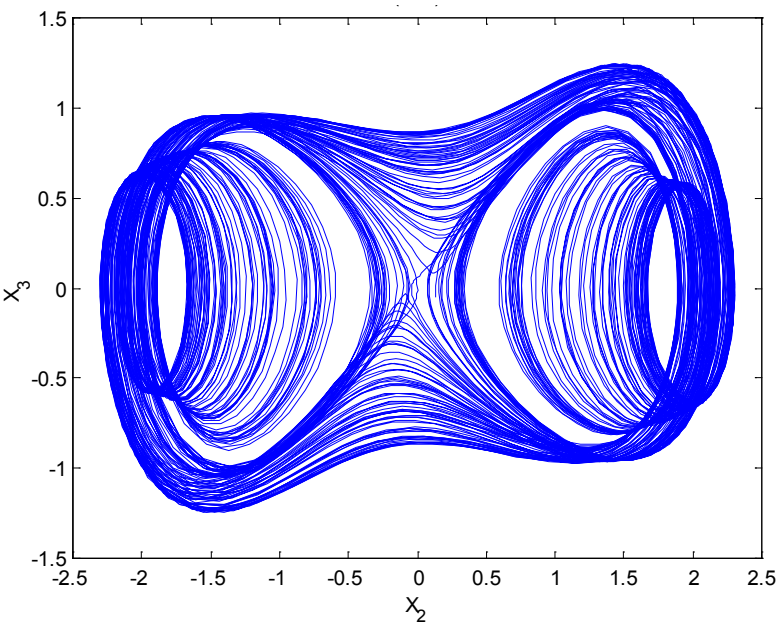

(b)

Figure 2. Uncontrolled simulations, (a) The chaotic time response and (b) State plane of the state variables $\mathrm{X}_{2}$ and $\mathrm{X}_{3}$ at Ko $=85,300$.

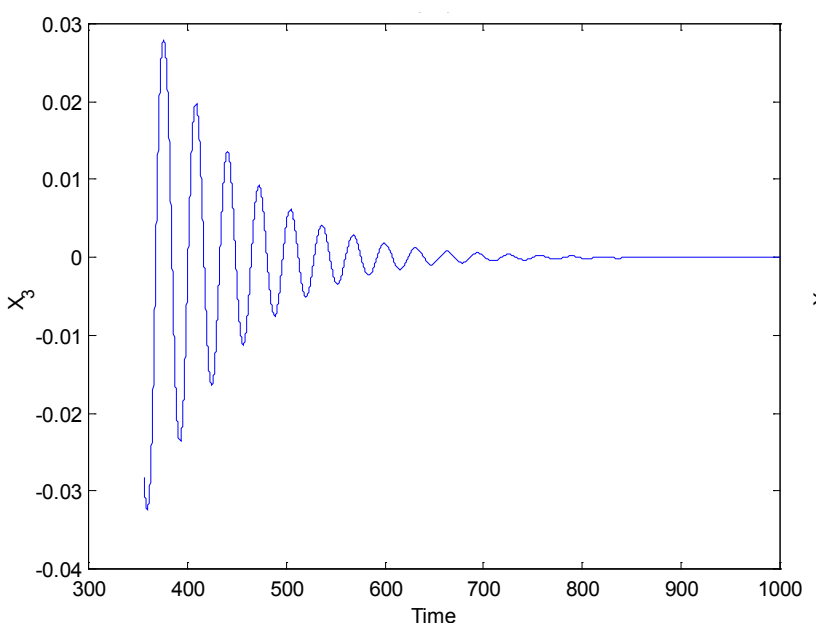

(a)

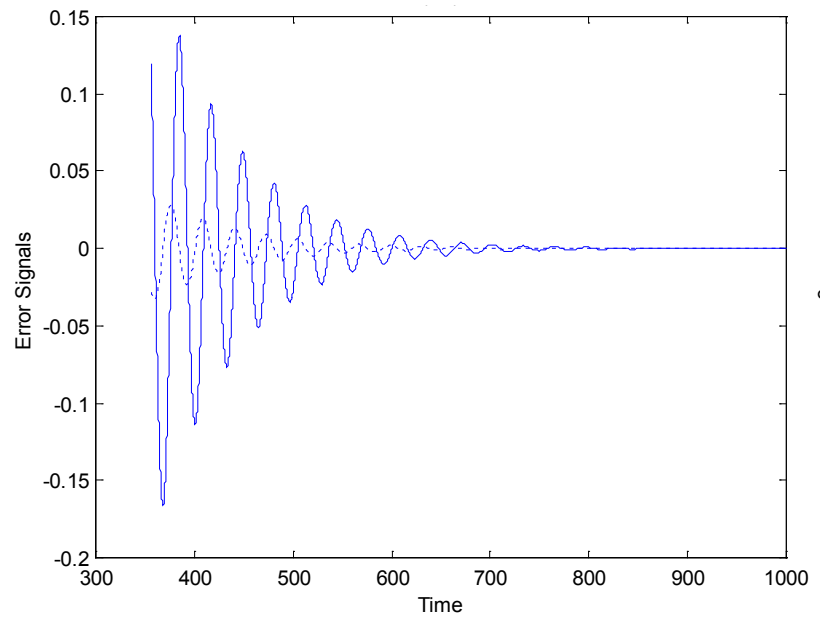

(c)

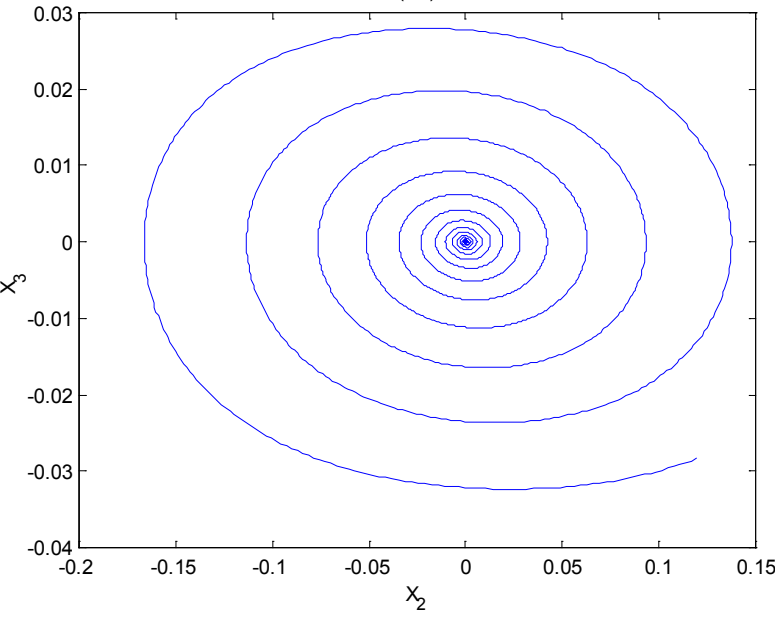

(b)

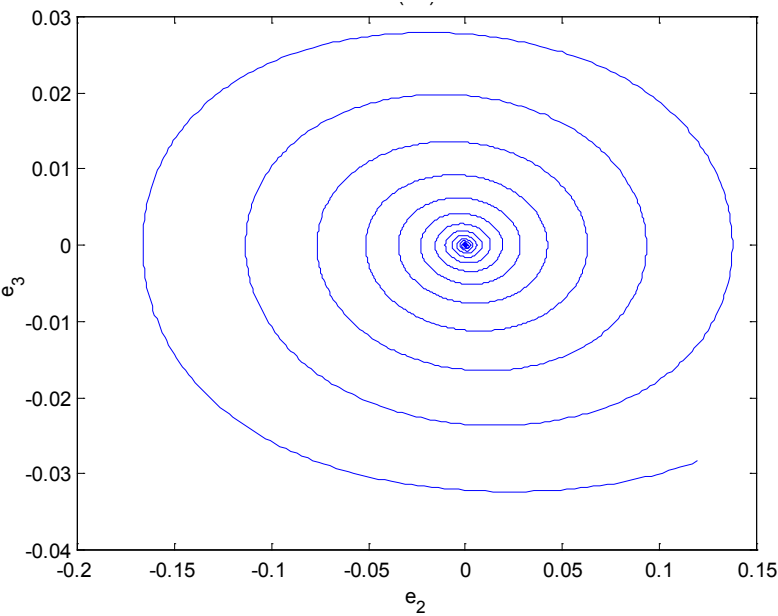

(d)

Figure 3. Controlled simulations [(a) Time history and (b) State plane] of the state variables $\mathrm{X}_{2}$ and $\mathrm{X}_{3}$, at $\mathrm{Ko}=85,300$. Controlled simulations [(c) Time history and (d) State plane) of the error signals $\mathrm{e}_{2}$ and $\mathrm{e}_{3}$, at Ko $=85,300$. 


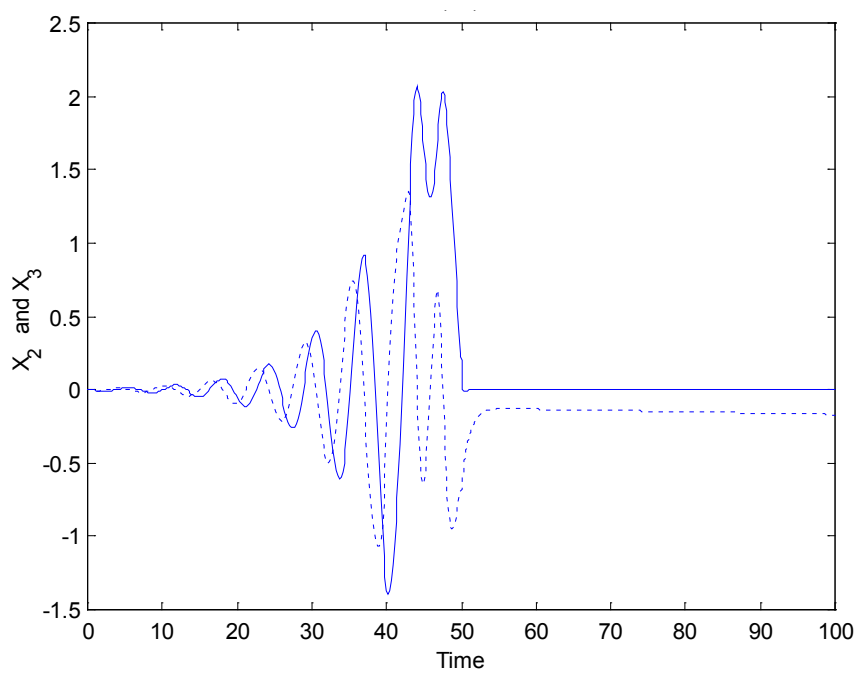

(a)

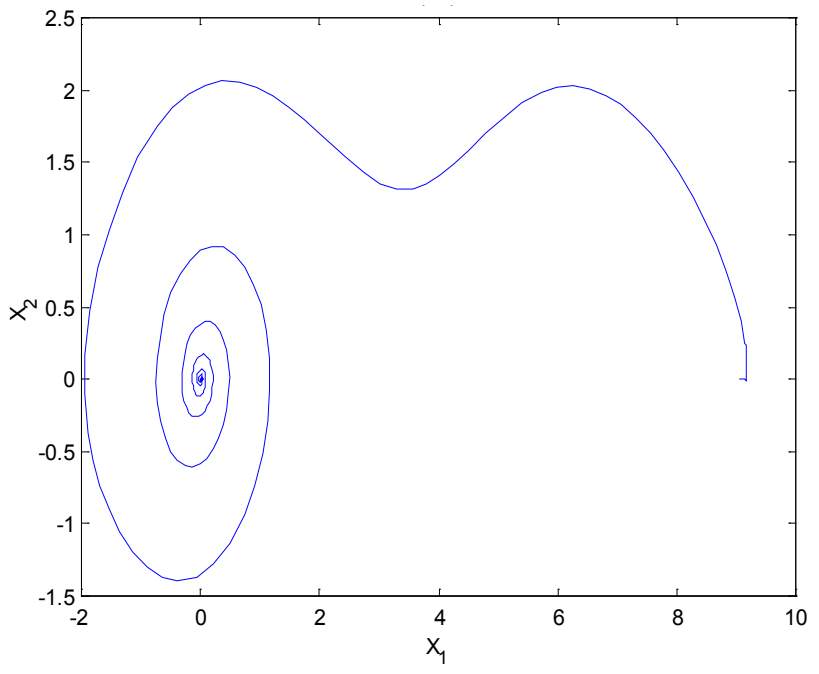

(b)

Figure 4. Applying the control signal after 50 seconds, (a) The time history and (b) The state plane of state variables $\mathrm{X}_{2}$ and $\mathrm{X}_{3}$, at Ko $=85,300$.

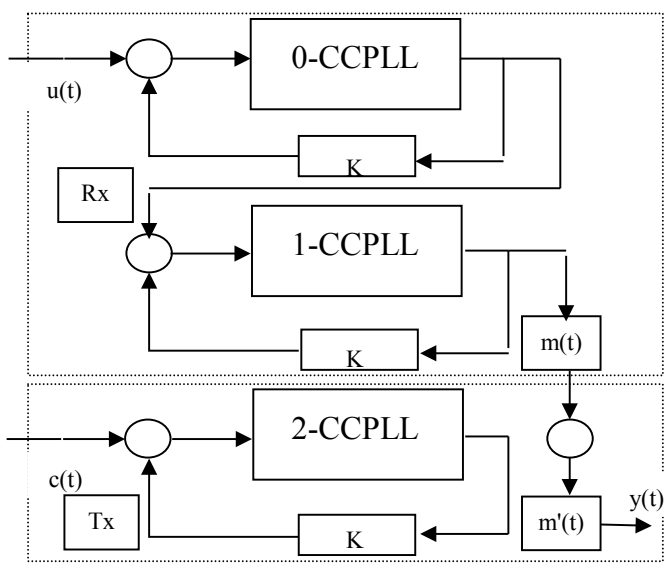

Figure 5. The block diagram of the closed phase locked loop model.

We initially start by driving the differential equations of the system. This is done by taking the receiving end containing 0 -CPLL and 1-CPLL to a chaotic state as represented in Equation (1),

$$
\dddot{\phi}_{o}+a \dddot{\phi}_{o}+b \cos \left(\phi_{o}\right) \ddot{\phi}_{o}+c \dot{\phi}_{o}+d \cos \left(\phi_{o}\right) \dot{\phi}_{o}-b \sin \left(\phi_{o}\right) \dot{\phi}_{o}^{2}+\sin \left(\phi_{o}\right)=\delta
$$

Next, we take the differential equation representing 1-CPLL is represented by Equation (2),

$$
\begin{aligned}
& \dddot{\phi}_{1}+a \ddot{\phi}_{1}+b \cos \left(\phi_{1}\right) \ddot{\phi}_{1}+c \dot{\phi}_{1}+d \cos \left(\phi_{1}\right) \dot{\phi}_{1}-b \cos \left(\phi_{o}\right) \dot{\phi}_{o}-d \cos \left(\phi_{o}\right) \dot{\phi}_{o} \\
& +b \sin \left(\phi_{o}\right) \dot{\phi}_{o}^{2}-b \sin \left(\phi_{1}\right) \dot{\phi}_{1}^{2}+\sin \left(\phi_{1}\right)-\sin \left(\phi_{o}\right)=0
\end{aligned}
$$

And for the transmission side 2-CPLL we have Equation (3),

$$
\begin{aligned}
& \dddot{\phi}_{2}+a \ddot{\phi}_{2}+b \cos \left(\phi_{2}\right) \ddot{\phi}_{2}+c \dot{\phi}_{1}+d \cos \left(\phi_{2}\right) \dot{\phi}_{2}-b \cos \left(\phi_{o}\right) \dot{\phi}_{o}-d \cos \left(\phi_{o}\right) \dot{\phi}_{o} \\
& +b \sin \left(\phi_{o}\right) \dot{\phi}_{o}^{2}-b \sin \left(\phi_{2}\right) \dot{\phi}_{2}^{2}+\sin \left(\phi_{2}\right)-\sin \left(\phi_{o}\right)=0
\end{aligned}
$$


We will introduce a set of variables to develop our model into a state variable set of space equations,

$$
x_{1}=\phi_{o}, x_{2}=\dot{\phi}_{o}, x_{3}=\ddot{\phi}_{o}, x_{4}=\phi_{1}, x_{5}=\dot{\phi}_{1}, x_{6}=\ddot{\phi}_{1}, x_{7}=\phi_{2}, x_{8}=\dot{\phi}_{2}, x_{9}=\ddot{\phi}_{2}
$$

Hence, our system's state space equations becomes,

$$
\begin{aligned}
\dot{x}_{1}= & x_{2} \\
\dot{x}_{2}= & x_{3} \\
\dot{x}_{3}= & \delta-a x_{3}-b \cos \left(x_{1}\right) x_{3}-c x_{2}-d \cos \left(x_{1}\right) x_{2}+b \sin \left(x_{1}\right) x_{2}^{2}-\sin \left(x_{1}\right) \\
\dot{x}_{4}= & x_{5} \\
\dot{x}_{5}= & x_{6} \\
\dot{x}_{6}= & -a x_{6}-b \cos \left(x_{4}\right) x_{6}-c x_{5}-d \cos \left(x_{4}\right) x_{5}+b \cos \left(x_{1}\right) x_{3}+d \cos \left(x_{1}\right) x_{2} \\
& -b \sin \left(x_{1}\right) x_{2}^{2}+b \sin \left(x_{4}\right) x_{5}^{2}+\sin \left(x_{1}\right)-\sin \left(x_{4}\right) \\
\dot{x}_{7}= & x_{8} \\
\dot{x}_{8}= & x_{9} \\
\dot{x}_{9}= & -a x_{9}-b \cos \left(x_{7}\right) x_{9}-c x_{8}-d \cos \left(x_{7}\right) x_{8}+b \cos \left(x_{1}\right) x_{3}+d \cos \left(x_{1}\right) x_{2} \\
& -b \sin \left(x_{1}\right) x_{2}^{2}+b \sin \left(x_{7}\right) x_{8}^{2}+\sin \left(x_{1}\right)-\sin \left(x_{7}\right)
\end{aligned}
$$

\section{Simulation and Analysis}

We have run the simulation in order to verify the synchronization within the communication system that is based on our CPLL model. These simulations resulted as expected that the synchronization depended on the initial conditions along with the parameters of our system. Figure 6 represents the results of the synchronization for both the transmission and receiver ends of our CPLL system. These are clearly shown that both initial conditions and parameters matched for both transmission and receiver ends.

We selected the phase errors $\Phi_{1}$ and $\Phi_{2}$ to be as the transmission end and receiver end respectively. Furthermore, we introduce an information sinusoidal signal and denote it by $\mathrm{m}(\mathrm{t})$ to be multiplied by the transmission phase error $\Phi_{1}$. As a result, Figure 7 represents the reconstructed receiver end signal that is synchronized at the output as indicated above.

As a result of a mismatch in the initial conditions for both ends of the transmission and receiver ends would indicate a non-synchronized signal as it is apparent in Figure 8. Thus, it is impossible at this stage to recover the information sinusoidal signal $\mathrm{m}(\mathrm{t})$, that was modulated through a chaotic carrier. Figure 9 shows the recovering sinusoidal signal that have failed to be recovered for the non synchronized original signal due to the mismatch in the initial conditions and parameter.

In general, we have proven that the chaotic system depends implicitly to the origination of a matched initial conditions and matched parameters of the complete system in study.

\section{Conclusion}

Our simulation and analysis indicate that the two chaotic outputs of identical 

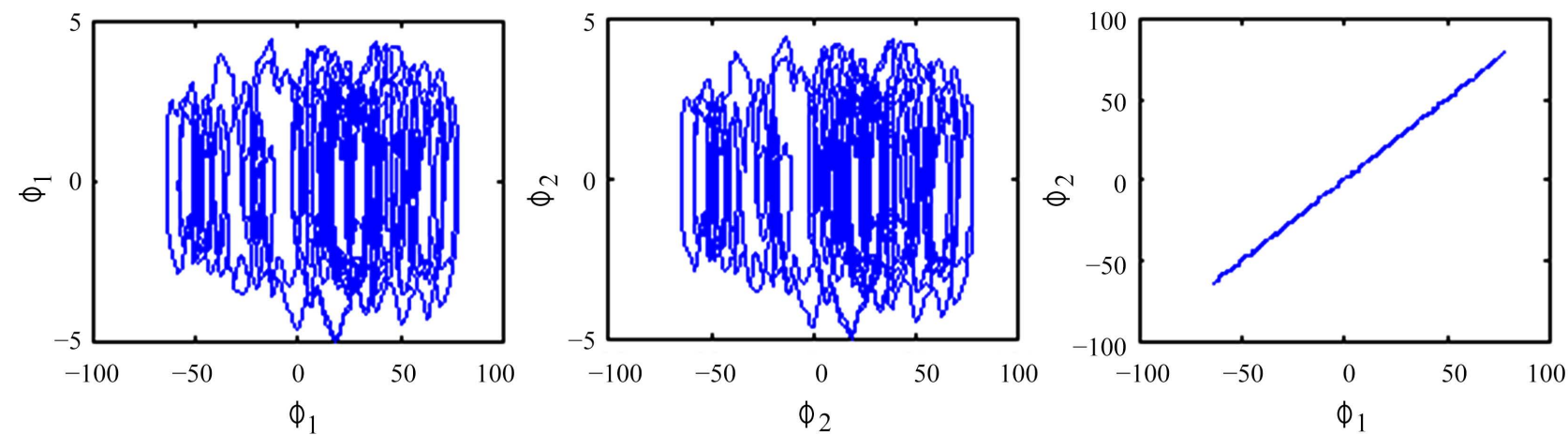

Figure 6. Results of the synchronization for both the transmission and receiver ends of our CPLL system.
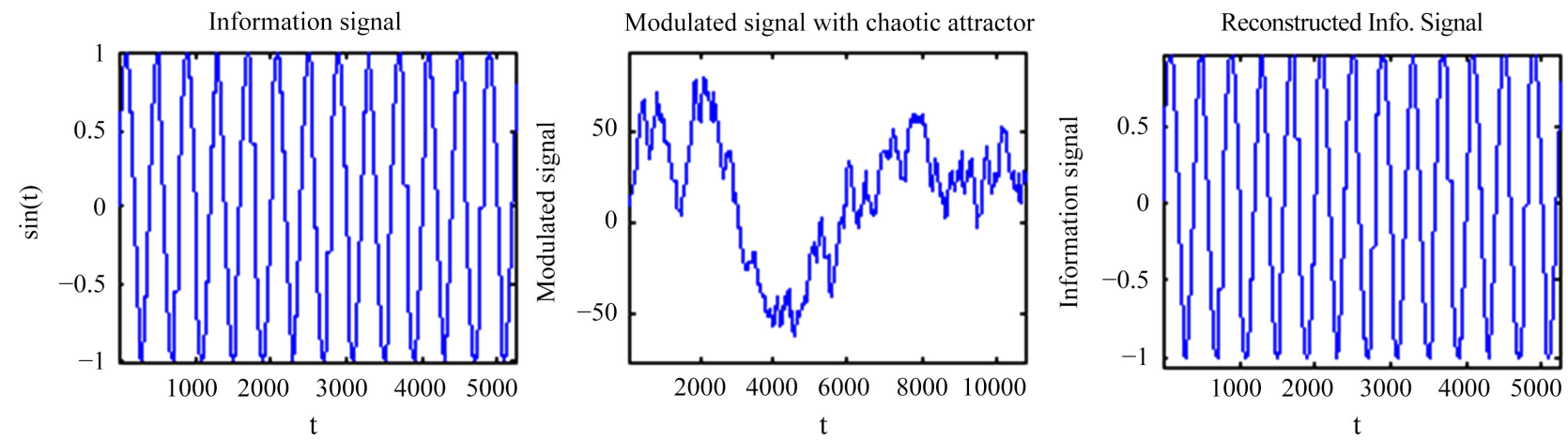

Figure 7. The reconstructed receiver end signal that is synchronized at the output.
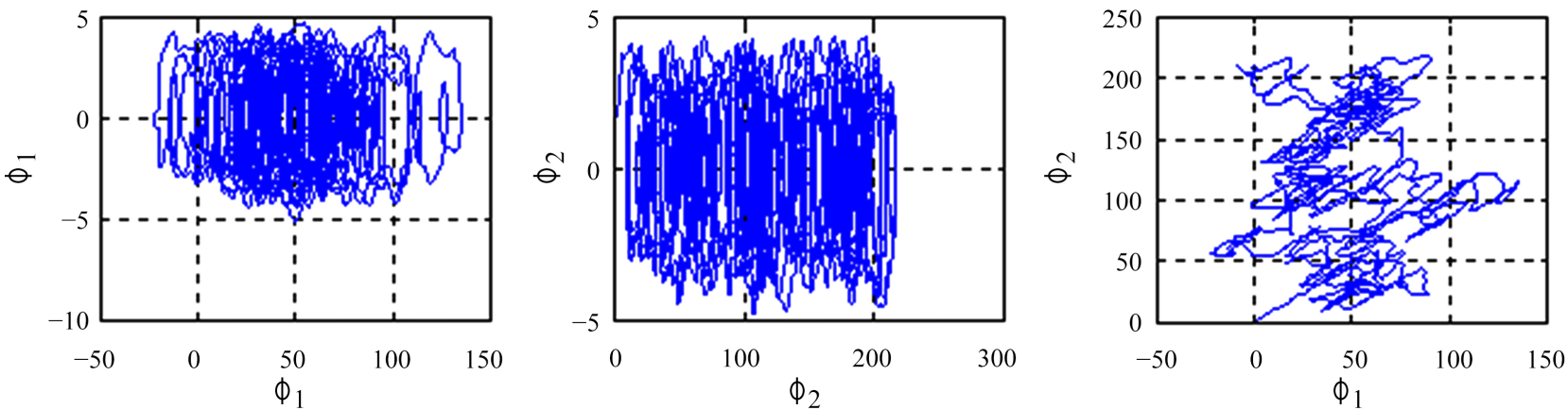

Figure 8. Non synchronization when a difference in the initial conditions exists.
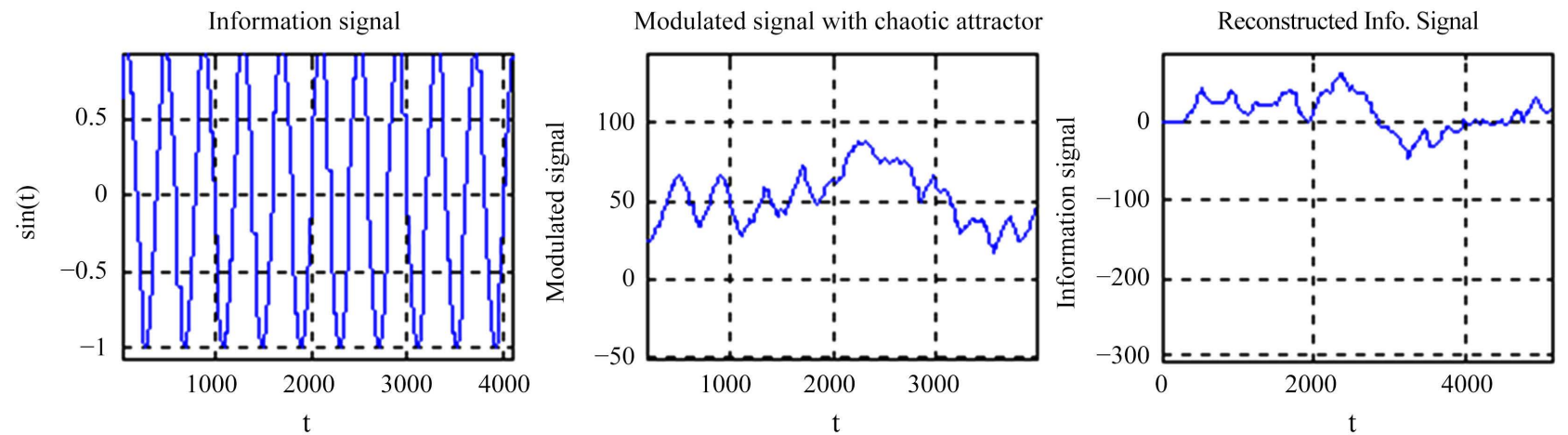

Figure 9. The recovering sinusoidal signal that has failed to be recovered for the non synchronized original signal. 
CPLLs, $\mathrm{V}_{\text {co }}$ would result in a common chaotic input synchronized sinusoidal signal. The initial conditions along with the original parameters matching as well as with both the transmission and receiver ends will result into a one to one synchronization system. It has also been proven that a small mismatch in the either the initial conditions or the original parameters would result in an impossible recovery of the information sinusoidal signal transmitted and received.

\section{References}

[1] Carroll, T.L. and Pecora, L.M. (1998) Synchronizing Hyperchaotic Volume-Preserving Maps and Circuits. IEEE Transactions on Circuits and Systems I-regular Papers, 45, 656-659. https://doi.org/10.1109/81.678482

[2] Pecora, L.M. and Carrol, L. (1990) Synchronization in Chaotic Systems. Physical Review Letters, 64, 821-824. https://doi.org/10.1103/PhysRevLett.64.821

[3] Pecora, L.M. and Carrol, T.L. (1991) Synchronizing Chaotic Circuits. IEEE Transactions on Circuits and Systems, 38, 453-456. https://doi.org/10.1109/31.75404

[4] Endo, T. and Chua, L.O. (1991) Synchronization of Chaos in Phase-Locked Loops. IEEE Transactions on Circuits and Systems, 38, 1580-1588. https://doi.org/10.1109/31.108517

[5] Harb, A. and Zohdy, M. (2002) Synchronization of Chaotic Systems Applied to Communications Systems. Conference Paper.

[6] Sato, A. and Endo, T. (1995) Experiments of Scure Communications via Chaotic Synchroniztion of Phase-Locked Loops. IEICE Transactions on Fundamentals of Electronics Communications and Computer Sciences, E78, 1286-1290.

[7] Carroll, T.L., Johnson, G.A. and Pecora, L.M. (1999) Parameter-Insensitive and Narrow-Band Synchronization of Chaotic Circuits. International Journal of Bifurcation and Chaos, 9, 2189-2196. https://doi.org/10.1142/S0218127499001619

[8] Wu, S. (1987) Chua's Circuit Family. Proceedings of the IEEE, 75, 1022-1032. https://doi.org/10.1109/PROC.1987.13847

[9] Salam, F. and Sastry, S. (1985) Dynamics of the Forced Josephson Junction Circuit: The Region of Chaos. IEEE Transactions on Circuit and Systems, 32, 784-796.

[10] Kennedy, M.P. and Chua, L.O. (1981) Van der Pol and chaos. IEEE Transactions on Circuits and Systems, 33, 974-980. https://doi.org/10.1109/TCS.1986.1085855

[11] Endo, T. and Chua, L.O. (1988) Chaos from Phase-Locked Loops. IEEE Transactions on Circuits and Systems, 35, 987-1003. https://doi.org/10.1109/31.1845

[12] Watada, K., Endo, T. and Seishi, H. (1998) Shilnikov Orbits in an Autonomous Third-Order Chaotic Phase-Locked Loop. IEEE Transactions on Circuits and Systems I: Fundamental Theory and Applications, 45, 979-983. https://doi.org/10.1109/81.721264

[13] Harb, B. and Harb, A. (2003) Chaos and Bifurcation in Third-Order Phase Locked Loop. Proceedings of the 35 th Southeastern Symposium on System Theory, Morgantown, WV, 18-18 March 2003. https://doi.org/10.1109/SSST.2003.1194549

[14] Harb, A., Zaher, A. and Zohdy, M. (2002) Nonlinear Recursive Backstepping Chaos Control, ACC-Alaska.

[15] Frey, D.R. (1993) Chaotic Digital Encoding: An Approach to Secure Communications. IEEE Transactions on Circuits and Systems, 40, 660-666. https://doi.org/10.1109/82.246168

[16] Jackson, E.A. (1991) Control of Dynamic Flows with Attractors. Physical Review A, 44, 4839-4853. https://doi.org/10.1103/PhysRevA.44.4839 